\title{
$X$ Herculis and TX Piscium: two cases of ISM interaction with stellar winds observed by Herschel ${ }^{\star}$
}

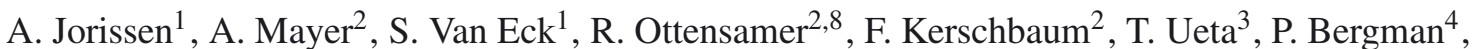 \\ J. A. D. L. Blommaert ${ }^{5}$, L. Decin ${ }^{5}$, M. A. T. Groenewegen ${ }^{6}$, J. Hron ${ }^{2}$, W. Nowotny ${ }^{2}$, H. Olofsson ${ }^{4}$, Th. Posch ${ }^{2}$, \\ L. O. Sjouwerman ${ }^{7}$, B. Vandenbussche ${ }^{5}$, and C. Waelkens ${ }^{5}$

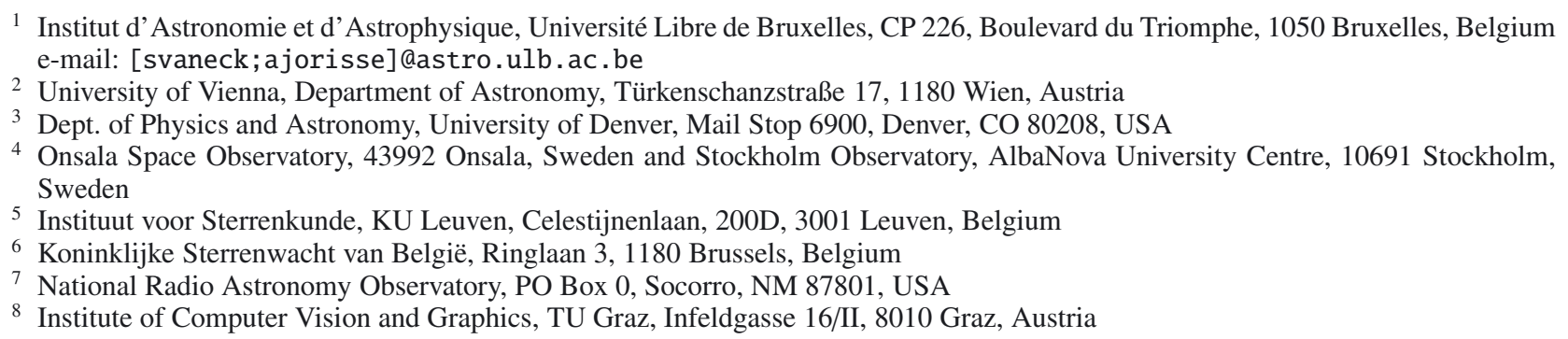

Received 19 March 2011 / Accepted 24 May 2011

\begin{abstract}
The asymptotic giant branch (AGB) stars X Her and TX Psc have been imaged at 70 and $160 \mu \mathrm{m}$ with the PACS instrument onboard the Herschel satellite, as part of the large MESS (Mass loss of Evolved StarS) guaranteed time key program. The images reveal an axisymmetric extended structure with its axis oriented along the space motion of the stars. This extended structure is very likely to be shaped by the interaction of the wind ejected by the AGB star with the surrounding interstellar medium (ISM). As predicted by numerical simulations, the detailed structure of the wind-ISM interface depends upon the relative velocity between star+wind and the ISM, which is large for these two stars (108 and $55 \mathrm{~km} \mathrm{~s}^{-1}$ for X Her and TX Psc, respectively). In both cases, there is a compact blob upstream whose origin is not fully elucidated, but that could be the signature of some instability in the wind-ISM shock. Deconvolved images of X Her and TX Psc reveal several discrete structures along the outermost filaments, which could be Kelvin-Helmholtz vortices. Finally, TX Psc is surrounded by an almost circular ring (the signature of the termination shock?) that contrasts with the outer, more structured filaments. A similar inner circular structure seems to be present in X Her as well, albeit less clearly.
\end{abstract}

Key words. ISM: general - stars: AGB and post-AGB - stars: mass-loss - infrared: stars - stars: carbon

\section{Introduction}

The MESS (Mass loss of Evolved StarS) guaranteed time key program (Groenewegen et al. 2011) observed with the PACS and SPIRE instruments onboard the Herschel Space Observatory a representative sample of evolved stars, including 78 asymptotic giant branch (AGB) stars and post-AGB stars with the aim of studying their circumstellar environments and mass-loss history by taking advantage of the good angular resolution offered by Herschel (with a point-spread function of 5'.7 full-width at half maximum at $70 \mu \mathrm{m})$.

A large variety of shapes (spherical, elliptical, detached, and axisymmetric shells) has been encountered by this program. A similar conclusion is reached for smaller angular scales $\left(1-10^{\prime \prime}\right)$ by the Plateau de Bure/Pico Veleta CO survey (Castro-Carrizo et al. 2007, 2010). In addition, Mid-IR and optical surveys (Meixner et al. 1999; Ueta et al. 2000) have shown that the shells exhibit at least axisymmetry by the end of the AGB phase. AGB stars with detached shells in the MESS sample have already been

\footnotetext{
* Herschel is an ESA space observatory with science instruments provided by European-led Principal Investigator consortia and with important participation from NASA.
}

discussed by Kerschbaum et al. (2010, 2011), and a synoptic paper is in preparation (Cox et al.). The present paper focuses on two spectacular cases, namely the O-rich star X Her and the C-rich star TX Psc, which show axisymmetric shells.

Extended asymmetric structures around AGB stars have been seen in different wavelength bands (which thus probe different regions of the circumstellar environment): in the IR by Young et al. (1993), Ueta et al. (2006, 2008, 2010), Ueta (2008), Geise et al. (2010), Ladjal et al. (2010), and Izumiura et al. (2011), in H I at $21 \mathrm{~cm}$ by Gardan et al. (2006), Matthews \& Reid (2007), Matthews et al. (2008), Libert et al. (2010), and Matthews et al. (2011), in the CO radio lines by Heske et al. (1989), Kahane \& Jura (1996), Libert et al. (2010), and CastroCarrizo et al. (2010), and in the UV by Martin et al. (2007). These asymmetries can be triggered by several causes:

- wind ejection in a binary system will shape a spiral stream, according to hydrodynamical simulations (Theuns \& Jorissen 1993; Theuns et al. 1996; Mastrodemos \& Morris 1999; Huggins et al. 2009). The clearest illustration thereof has been observed in the proto-planetary nebula AFGL 3068 (Mauron \& Huggins 2006; Morris et al. 2006); 
- asymmetric mass loss in single stars, as predicted by dustdriven wind models for red giants with cool spots (Reimers 2005) and 3D models of carbon stars (Freytag \& Höfner 2008), may produce an off-centred nebula. Such an asymmetric mass loss may also impart a kick on the forming white dwarf, and evidence thereof has been reported in globular clusters (Heyl 2007a,b, 2008; Davis et al. 2008; Fregeau et al. 2009);

- Matt et al. (2000) demonstrated that a stellar dipole magnetic field can focus an initially isotropic wind toward the equatorial plane, thus producing an equatorial overdensity, but Soker (2005) pointed out that in cases where the magnetic field plays a global role in the shaping, a binary companion is necessary to maintain the field;

- interaction between the stellar wind and the interstellar medium (ISM), which is the topic of the present paper.

For X Her and TX Psc, there are strong indications that the interaction of their wind with the ISM shapes their axisymmetric shell. First evidence of this kind of interaction have been seen in planetary nebulae (PNe), in the form of axisymmetric morphologies oriented along the space motion of the central star (e.g., Jacoby 1981; Reynolds 1985; Krautter et al. 1987; Borkowski et al. 1990; Dgani \& Soker 1998, and references therein). Among non-AGB stars, far-IR parabolic arcs attributable to bow shocks have been discovered around $\alpha$ Ori from IRAS data by Stencel et al. (1988), and later confirmed by AKARI data (Ueta et al. 2008). Runaway OB stars are another class of stars around which bow shocks are frequent (Gull \& Sofia 1979; van Buren \& McCray 1988; van Buren 1993; van Buren et al. 1995).

Gardan et al. (2006), Ueta et al. (2006), and Martin et al. (2007) mentioned the possibility of wind - ISM interaction in relation to AGB mass loss, although the wind velocity is very small $\left(<20 \mathrm{~km} \mathrm{~s}^{-1}\right)$ in comparison with $\mathrm{PNe}$ or OB stars $\left(\sim 1000 \mathrm{~km} \mathrm{~s}^{-1}\right)$ and shock structures seemed therefore unlikely.

In this scenario, the AGB wind is slowed down as it sweeps up ISM material. The swept-up material forms a density enhancement that continues to expand because of the internal pressure. The thermal emission of the dust at the shock interface between the stellar wind and the ISM has been detected in the far IR (Ueta et al. 2006, 2009), or directly in the X/UV, as for the bow shock associated with the wind of $o$ Ceti and detected by GALEX (Martin et al. 2007). Images obtained with Spitzer and AKARI have confirmed such an interaction scenario for the targets R Hya, R Cas, and $o$ Cet (Ueta et al. 2006; Ueta 2008; Ueta et al. 2010). In RX Lep, Libert et al. (2008) found an H I tail extending $0.5 \mathrm{pc}$ southward, as already suggested for Mira, RS Cnc, and other sources detected in H I (Gardan et al. 2006; Matthews \& Reid 2007; Martin et al. 2007; Matthews et al. 2008; Libert et al. 2010). Sahai \& Chronopoulos (2010) presented ultraviolet GALEX images of IRC+10 216 (CW Leo) revealing for the first time its bow shock, which is also visible in Herschel PACS and SPIRE infrared images (Ladjal et al. 2010).

\section{Basic data of the targets}

\section{1. $\times \mathrm{Her}$}

$\mathrm{X}$ Her is an oxygen-rich M8III semi-regular variable (SRV) of period $95 \mathrm{~d}$ and with a long secondary period of $746 \mathrm{~d}$ (Hinkle et al. 2002). From a radial-velocity monitoring of the $\mathrm{CO}$ lines at $1.6 \mu \mathrm{m}$, the same authors discovered a period of $660 \mathrm{~d}$. That all six but one among the SRV stars monitored by Hinkle et al. have similar eccentricities and longitudes of periastron casts doubts

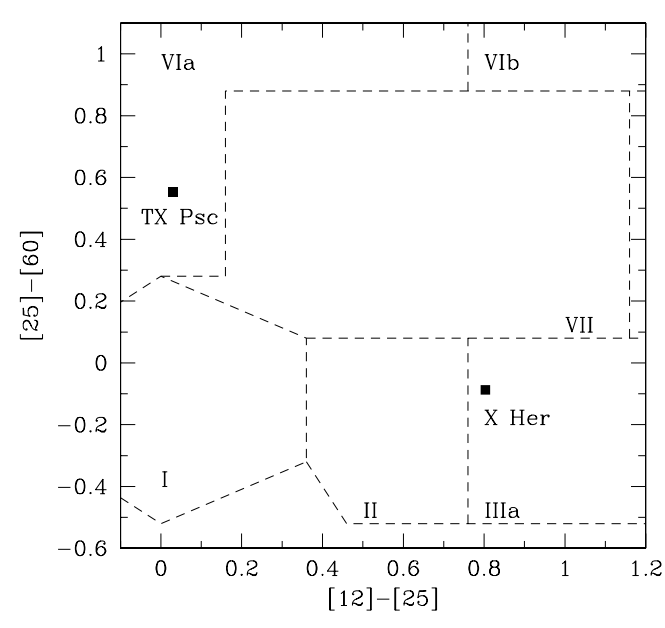

Fig. 1. Location of X Her and TX Psc in the IRAS colour-colour diagram, along with van der Veen \& Habing (1988) regions.

on the orbital origin of the observed velocity variations. The IRAS Low Resolution Spectrum of X Her was classified as E (i.e., silicate emission) by Kwok et al. (1997), and as SE6t (i.e., showing a $13 \mu \mathrm{m}$ feature, most probably due to Al-rich oxide dust, and strong amorphous silicate bands) by Sloan et al. (1996, 2003). Its position in region IIIa of the IRAS colour-colour diagram shown in Fig. 1 is consistent with the LRS class referring to a thick dust shell. From a model fit to the IRAS data, Young et al. (1993) found X Her to be extended to a radius of $6.2^{\prime}$ at $60 \mu \mathrm{m}$; however, the published data (their Fig. 11) revealed a much narrower profile, with a radius of the order of $2^{\prime}$, and some substructure, which could possibly be attributed to the nearby pair of background galaxies UGC 10156a and b (see Fig. 4 below).

\section{2. $T X P S C$}

TX Psc is one of the brightest and nearest optical carbon stars and has been extensively observed from the visual to $\mathrm{mm}$ waves. The General Catalogue of Variable Stars (GCVS) indicates a variability type $\mathrm{Lb}$, but an average period of $224 \mathrm{~d}$ was found by Wasatonic $(1995,1997)$, indicating that SRa/b may be more appropriate. The photometric variability in the $V$ band is of moderate amplitude $(0.4$ mag peak to peak in the $V$ band; see Fig. 2). However, ten new 2009-2010 observations with the HERMES/MERCATOR spectrograph (Raskin et al. 2010) spread over 450 days show large and regular variations in the radial velocity from 8 to $15 \mathrm{~km} \mathrm{~s}^{-1}$ (Fig. 2); these radial velocities were derived by correlating the observed spectrum with a template mask containing 3100 lines covering the spectral range 476-654 $\mathrm{nm}$. Earlier velocity measurements by Barnbaum \& Hinkle (1995) confirm this amplitude range, but are too sparsely sampled to reveal any underlying regularity. Simultaneous AAVSO photoelectric measurements confirm the photometric period of about $220 \mathrm{~d}$. Given that the regular velocity variations occur on a period substantially longer than the SRa/b period of $224 \mathrm{~d}$, these velocity variations cannot be interpreted as a manifestation of a pulsating atmosphere [see Lebzelter et al. (2001) for examples of a similar situation for radial velocities derived from $4 \mu \mathrm{m}$ lines]. They could either be an indication of the possible binary nature of TX Psc, or yet another 


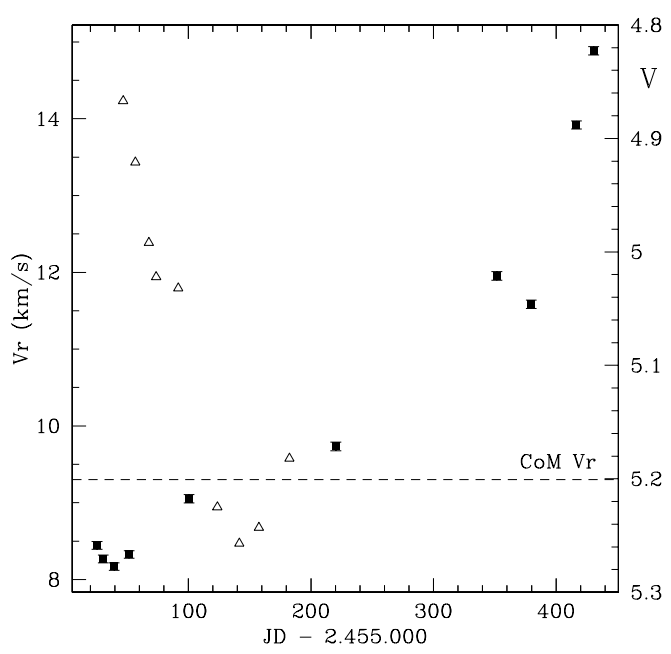

Fig. 2. Radial velocities of TX Psc obtained with the HERMES spectrograph and an Arcturus cross-correlation mask (black squares and left scale), along with AAVSO $V$ magnitudes (open triangles and right scale). The horizontal dashed line is the heliocentric centre-of-mass $(\mathrm{CoM})$ velocity from the $\mathrm{CO} J=1-0$ radio line (Barnbaum \& Hinkle 1995, and references therein).

example of the so-called long-secondary periods encountered in several long-period variables (including X Her; Hinkle et al. 2002; Nicholls et al. 2009) whose origin is still unclear. The radial-velocity monitoring is being pursued to clarify the origin of these variations. In the absence of any firm indication of the binary nature of TX Psc, we do not consider that possibility any further in this paper, especially since it may just be of a similar (non-orbital) nature as that observed for X Her and discussed above.

Although the IRAS low resolution spectrum of TX Psc has been classified as $\mathrm{S}$ (i.e., featureless, stellar-like) by Kwok et al. (1997), its $60 \mu \mathrm{m}$ flux is clearly indicative of cool dust, TX Psc falling in region VIa of the IRAS colour-colour diagram (Fig. 1), which is the locus of stars with detached dust shells. Young et al. (1993) indeed found TX Psc to be extended with a radius of roughly $3^{\prime}$ at $60 \mu \mathrm{m}$. From a fit to the near-IR and IRAS spectral energy distribution, Eriksson et al. (1986) have derived a dust temperature of $125 \mathrm{~K}$, but a more elaborate study by Wirsich (1991) suggests that the dust temperature decreases from $1155 \mathrm{~K}$ at 2.25 stellar radii to $410 \mathrm{~K}$ at 30 stellar radii.

\subsection{Kinematical data}

Proper motions, radial velocities, and parallaxes for X Her and TX Psc are listed in Table 1. For all calculations, we used the revised Hipparcos data by van Leeuwen (2007), and the systemic velocity derived from radio observations, which is consistent with the radial velocity derived from optical lines (Table 1). The space velocity is computed as follows. First the components $(U, V, W)$ of the spatial heliocentric velocity in Galactic coordinates are computed following Johnson \& Soderblom (1987), using the proper motion, the distance, and the radial velocity. The sources for these quantities used to compute the space velocity are given in Table 1 . The solar motion $(U, V, W)_{0}=$ $(11.10,12.24,7.25) \mathrm{km} \mathrm{s}^{-1}$ (Schönrich et al. 2010) is then added, and the $\alpha, \delta$ components of the space velocity, thus corrected from the solar motion, are then easily calculated by reversing
Table 1. Basic kinematical data for X Her and TX Psc.

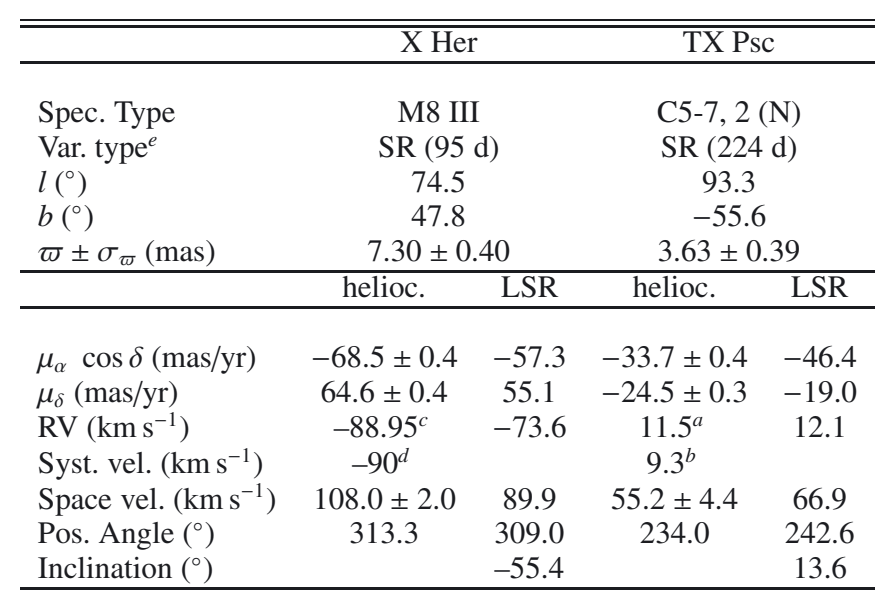

Notes. Parallaxes and proper motions are from van Leeuwen (2007). The column labelled "LSR" provides the kinematical data corrected from the solar motion.

References. ${ }^{(a)}$ Average HERMES velocity. ${ }^{(b)} \mathrm{CO} J=1-0$ heliocentric velocity from Barnbaum \& Hinkle (1995) and references therein. ${ }^{(c)}$ Famaey et al. (2005). ${ }^{(d)}$ Kahane \& Jura (1996). ${ }^{(e)}$ GCVS.

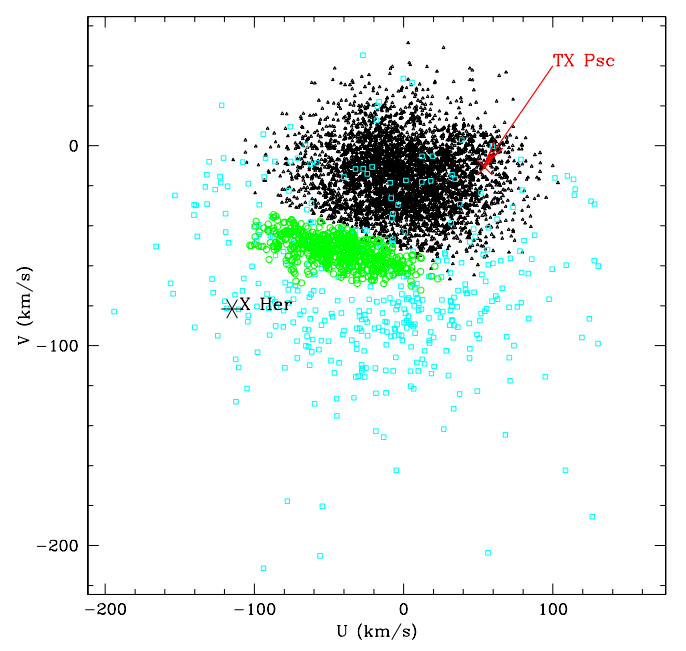

Fig. 3. Location of X Her and TX Psc in the $U V$ diagram, with data for $\mathrm{K}$ and M giants taken from Famaey et al. (2005). Black triangles denote stars belonging to the background velocity ellipsoid, (cyan) open squares to high-velocity stars, and (green) open circles to Herculesstream stars.

the procedure. In Table 1, the column labelled "LSR" provides all quantities corrected for the solar motion.

The location of X Her and TX Psc and in the $U V$ diagram is shown in Fig. 3, which reveals that X Her belongs to the group flagged as "high-velocity stars" by Famaey et al. (2005). TX Psc, in contrast, happens to fall in the background ellipsoid.

\section{Herschel data analysis}

\subsection{Observations and data reduction}

The images of X Her (Fig. 4) and TX Psc (Fig. 5) that we present here were obtained by the Photodetector Array Camera and Spectrometer (PACS) of Herschel on 2009 December 20 and 21, respectively, and are part of the MESS guaranteed time key programme. Details of the MESS programme can be found 

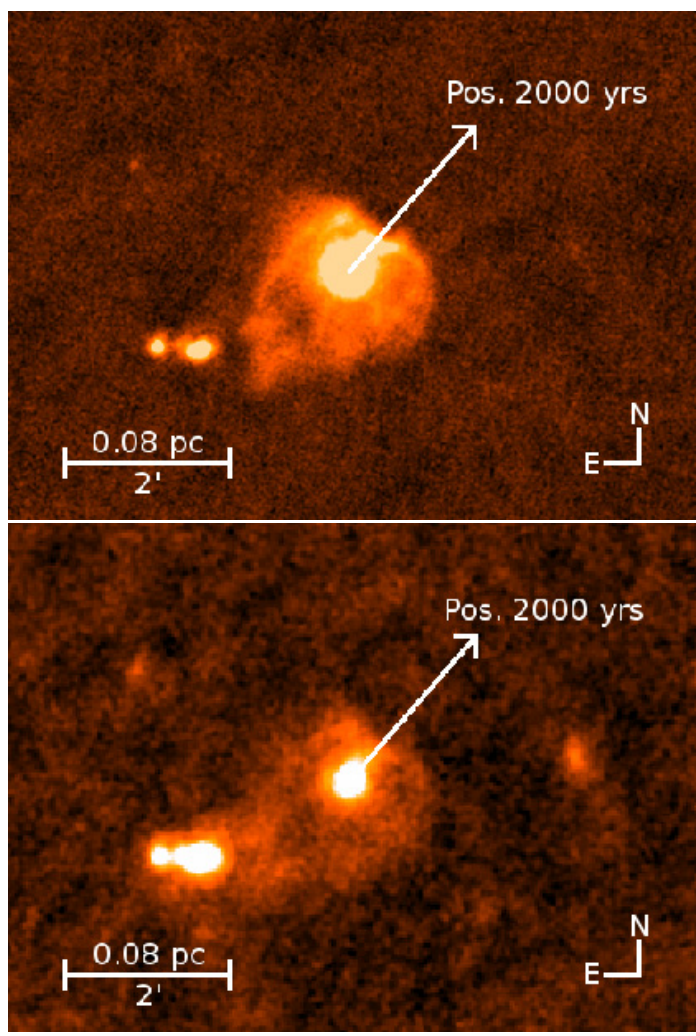

Fig. 4. PACS images of $X$ Her. The upper image is at $70 \mu \mathrm{m}$, the lower at $160 \mu \mathrm{m}$. The arrows indicate the direction of the proper motion and the position $2000 \mathrm{yrs}$ in the future. The two bright dots on the lower left side are a pair of galaxies (UGC 10156a and b). The faint source west of X Her is the galaxy MCG+08-29-036.

in Groenewegen et al. (2011), who describe as well the basic data processing and reduction with the Herschel Interactive Processing Environment (HIPE) that was used for producing Figs. 4 and 5. All images were oversampled by a factor of 3.2, which results in a sampling of 1 pixel per arcsec at $70 \mu \mathrm{m}$ and 1 pixel per 2 arcsec at $160 \mu \mathrm{m}$, to be compared with the $F W H M$ of 5.'7 and 11'. 4 at those wavelengths, respectively. Figure 6 presents deconvolved images of X Her and TX Psc. More details of the deconvolution methods used to produce these images can be found in Ottensamer et al. (2011).

\subsection{Structure and dynamical age of the circumstellar nebula}

The images of X Her (Figs. 4 and 6) and TX Psc (Figs. 5 and 6) reveal a wealth of features that we now discuss.

For both X Her and TX Psc, there is a noteworthy clump in the direction of the space motion (Fig. 6 and bottom right panel of Fig. 10). For TX Psc, there is also a ring around the star, which is clearly visible in the across scan cut (upper panel of Fig. 10). This ring is not an artefact of the deconvolution process, since it is also visible in the non-deconvolved image (Fig. 5). Moreover, a similar circular extended (filled) structure is also visible in the $70 \mu \mathrm{m}$ image of X Her (Fig. 6). TX Psc is close to the centre of this ring (the upstream radius is the same as the downstream radius; see Fig. 10). The circular symmetry of this feature is a clear indication that the mass loss from which it originates was isotropic, at variance with the situation currently prevailing closer to the star, where a bipolar outflow is observed (see Sect. 4). Moreover, the prominent nature of this ring on the PACS images may indicate that it actually corresponds to
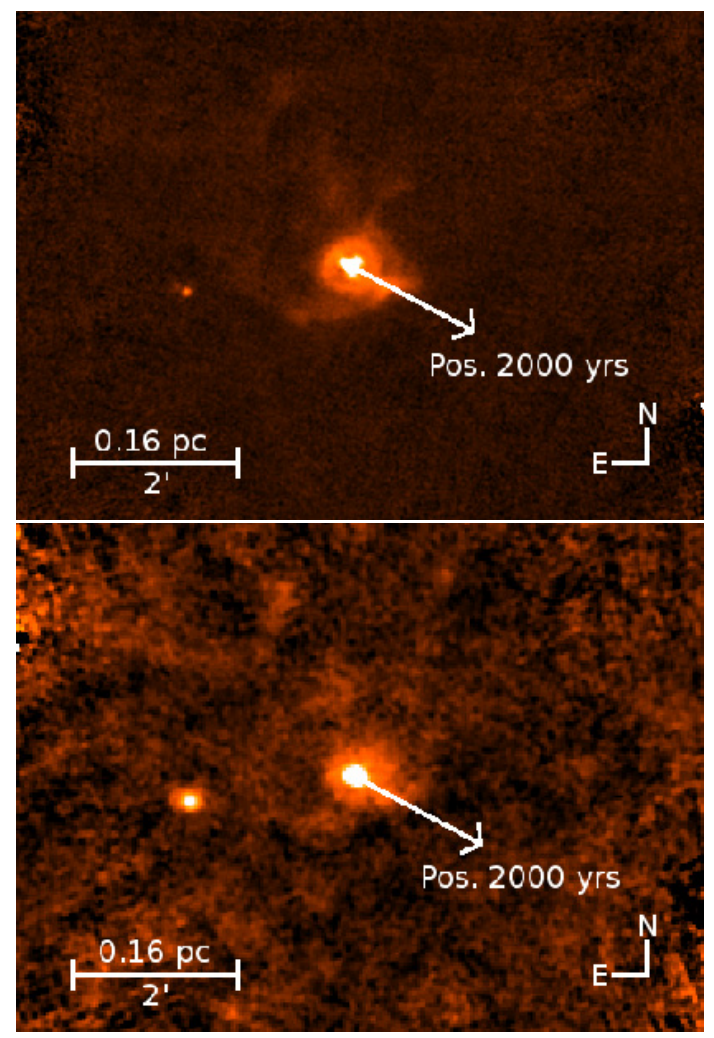

Fig. 5. Same as Fig. 4 for TX Psc. The bright source east of the star is an uncatalogued galaxy.

the termination shock, where the reverse shock from the windISM interface is facing the freely expanding stellar wind, although the available data offer so far no additional support of this hypothesis.

The dynamical ages of these features may be estimated as follows. The inner ring is at a distance of 17" from TX Psc downstream, which corresponds to a kinematic age of about 2100 yrs (Fig. 10) for an assumed wind velocity $v_{\mathrm{w}}=$ $10.5 \mathrm{~km} \mathrm{~s}^{-1}$ (Olofsson et al. 1993), neglecting any possible inclination on the plane of the sky. The blob in the direction of the space motion is at a distance of $29^{\prime \prime}$, corresponding to a kinematic age of $3610 \pm 410$ yrs. The wind-ISM interface limits the possibility of looking further back into the mass-loss history.

The same procedure and assumptions were applied to the heart-shaped X Her. With a wind velocity $v_{\mathrm{w}}=6.5 \mathrm{~km} \mathrm{~s}^{-1}$ (González-Delgado et al. 2003), the bow shock at a distance of $28^{\prime \prime}$ from the star has a minimum kinematic age of $2800 \pm 180 \mathrm{yrs}$ and the end of the tail at $112^{\prime \prime}$ downstream has a (minimum) age of $11200 \pm 680 \mathrm{yrs}$ (Fig. 9).

Another structural feature in TX Psc deserves discussion. A compact feature (representing $2 \%$ of the flux in the $K$ band and located upstream, at a position angle of $241^{\circ}$ ) was detected by the COME-ON+ observations of Cruzalèbes et al. (1998), but at a distance of only 0.35 from the primary source, thus well inside the ring described above. Although, at first sight, one would be tempted to relate this blob observed close to the star with the one observed in the PACS $70 \mu \mathrm{m}$ image discussed above, they cannot be caused by the same physical phenomenon (such as the hydrodynamical instabilities discussed later in the present section), because the COME-ON+ secondary source is located 

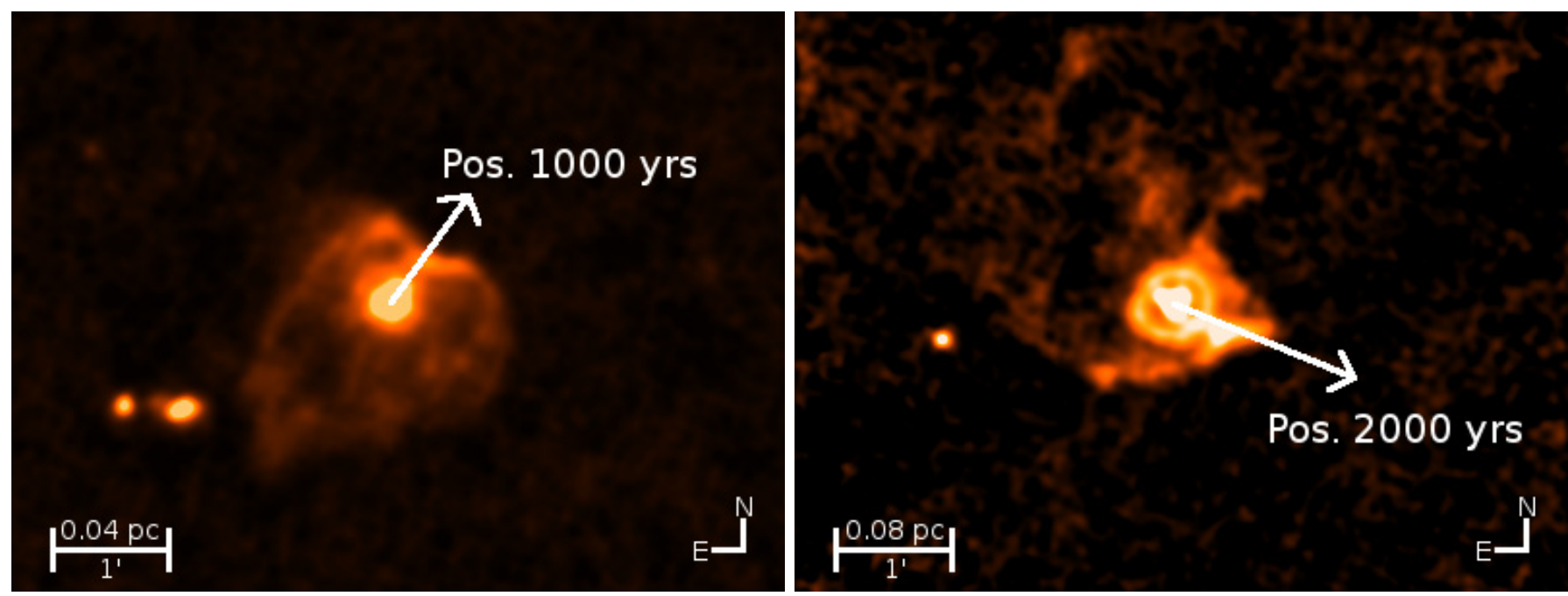

Fig. 6. Deconvolved PACS images of X Her (left) and TX Psc (right), both in the blue channel at $70 \mu \mathrm{m}$. The arrows indicate the direction of the proper motion and the position 1000 or 2000 yrs in the future. North is up and east is to the left.

within $^{1}$ the circular ring observed around TX Psc at a distance of about $17^{\prime \prime}$. As discussed earlier, structures within this ring cannot be caused by the interaction with the ISM. Nevertheless, the inner clump is observed at the same position angle as that corresponding to the space motion. Another puzzle, possibly related to the former, is the correlation existing between the space motion and the orientation of the bipolar outflows observed around both X Her (where it is almost exactly perpendicular to the space motion) and TX Psc (where it seems to be parallel with the space motion). The properties of the bipolar outflow are discussed further in Sect. 4. Finally, it is rather surprising that the morphology of the far-IR nebula does not bear any signature of the inner bipolar outflow, especially in the case of X Her where it is perpendicular to the space motion.

Comerón \& Kaper (1998) illustrated the wide variety of bow-shock structures that may arise from the interaction of the stellar wind with the ISM. They stressed the importance of the wind parameters (density and velocity), the star's velocity, and the ISM density. These parameters in turn fix the cooling efficiency of the gas, which appears to be an essential parameter in fixing the structure of the wind - ISM interface (Soker et al. 1991; van Buren 1993; Comerón \& Kaper 1998): if the cooling is efficient ${ }^{2}$, the gas can be compressed to high densities, and the shocked layer remains very thin $^{3}$. This situation is described well by the isothermal approximation. On the contrary, if cooling is inefficient, the high temperature of the gas limits the compression factor, which for the limiting case of no cooling, would be equal to 4 (abiabatic shock; Comerón \& Kaper 1998). In this situation, the shocked layer is thick. Different instabilities

\footnotetext{
1 It is in principle possible that this secondary source is actually located outside the circular ring, but seen inside only in projection. This would mean that the secondary source is then located almost along the line of sight, at a distance larger than about $4700 \mathrm{AU}\left(=17^{\prime \prime} / 0{ }^{\prime}\right.$.0036) from the star. In the absence of any other argument supporting this view, this possibility will not be considered further in this paper.

2 See Soker et al. (1991) for a basic description of the relation between the wind/ISM properties and the cooling efficiency. Dense gas generally gives rise to efficient cooling.

3 Wilkin fitting - geometrical fitting of the bow shock shape based on the analytical formulation by Wilkin (1996), which will be attempted in Sect. 3.4 - applies to the thin-shell approximation.
}

are encountered in thin and thick shocks (see Comerón \& Kaper 1998, for a review).

In thin shells, Dgani et al. (1996b,a) studied the so-called transverse acceleration instability, which occurs as a consequence of the acceleration in the flow normal to the shock caused by its curved surface. Another instability appearing under the same conditions is the non-linear thin-shell instability, which arises from the shear in shock-bounded slabs produced by deviations from equilibrium (Vishniac 1994; Blondin \& Marks 1996). If there is a shear along the surface of the bow shock, KelvinHelmholtz instabilities will develop as well. Numerical simulations relative to bow shocks with thin shells were performed by Soker et al. (1991), Dgani et al. (1996b), Comerón \& Kaper (1998), and Blondin \& Koerwer (1998).

If the shocked material cools rather inefficiently, a thick shell will form, which will be subject to other types of instabilities, mostly the Rayleigh-Taylor (R-T) instability, because the densities of the gas entering from either side of the shock will not match in the absence of cooling. When two fluids of different densities are penetrating each other, $\mathrm{R}-\mathrm{T}$ instabilities ${ }^{4}$ manifest themselves in the form of mushroom- and finger-shaped protuberances of the lighter fluid into the heavier one. Soker et al. (1991) demonstrated that R-T instabilities occur when a stellar wind or planetary nebula shell is colliding with a low-density ISM with a high relative velocity $\left(v_{*, \text { ISM }}>100 \mathrm{~km} \mathrm{~s}^{-1}\right)$, leading to a fragmentation of the shell. A bump in the upstream direction, attributed to a R-T instability developing at an early stage of the wind - ISM interaction, is seen for instance in the planetary nebula NGC 40 (Martin et al. 2002). At a later stage, as the shell fragments, Kelvin-Helmholtz $(\mathrm{K}-\mathrm{H})$ instabilities appear as a sequence of vortices peeling off the bow shock and moving downstream (Wareing et al. 2007a,b).

The nebulae around X Her and TX Psc show very unusual features, such as the upstream blob and clumps visible along what seems to be a bow shock. Investigations of the shape

\footnotetext{
4 A related instability is the Richtmyer-Meshkov instability (e.g., Needham 2010), which develops when a shock wave hits the interface of two fluids with different densities, such as when the new shell of an expanding wind bubble hits the already established bow shock. This may happen as AGB stars lose mass in a succession of discrete events, as suggested by the successive shells observed around e.g. the carbon Mira IRC + 10216 (Mauron \& Huggins 2000).
} 


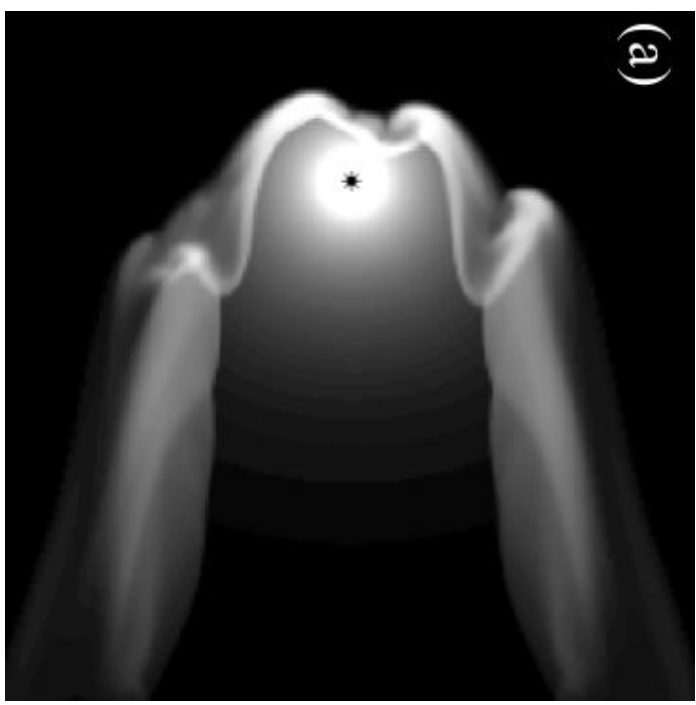

Fig. 7. The gas density in case E simulation of Wareing et al. (2007a) (corresponding to $v_{*, \mathrm{ISM}}=125 \mathrm{~km} \mathrm{~s}^{-1}, n_{\mathrm{H}}=2 \mathrm{~cm}^{-3}$, and a mass-loss rate of $5 \times 10^{-6} M_{\odot} \mathrm{yr}^{-1}$ ), which reveal the formation of vortices in the wake of the AGB star. This stage corresponds to the end of the AGB phase, and resembles the X Her nebula (compare with Fig. 6, remembering that the viewing angle is different, since the space motion of $\mathrm{X}$ Her is inclined by $55^{\circ}$ with respect to the plane of the sky). Reprinted from Wareing et al. 2007, MNRAS, 382, 1233, with permission from John Wiley \& Sons.

of the bow shock have been made using hydrodynamical simulations by Blondin \& Koerwer (1998), Comerón \& Kaper (1998), Villaver et al. (2003), and Wareing et al. (2007a). The Case E simulation of the last set of authors (corresponding to $v_{*, \mathrm{ISM}}=125 \mathrm{~km} \mathrm{~s}^{-1}, n_{\mathrm{H}}=2 \mathrm{~cm}^{-3}$, and a mass-loss rate of $5 \times 10^{-6} M_{\odot} \mathrm{yr}^{-1}$; see Fig. 7), at stage a (corresponding to the end of the AGB phase), looks very similar ${ }^{5}$ to the X Her nebula (compare Figs. 6 and 7), since it combines an upstream shock surface bent towards the star, and K-H vortices in the downstream wake. Interestingly, the $90 \mathrm{~km} \mathrm{~s}^{-1}$ LSR space velocity of X Her (Table 1) is similar to the velocity used in the Wareing et al. simulation, but the current mass-loss rate is a factor of at least 30 lower than the Wareing value (see Sect. 4.1). On the other hand, the TX Psc nebula bears similarities (especially the strong upstream blob, and the inhomogeneous bow) with the large Mach number (10) and isothermal simulations of Blondin \& Koerwer (1998; compare Figs. 6 and 8). A large Mach number is indeed expected in the present situation, since the X Her space velocity is $67 \mathrm{~km} \mathrm{~s}^{-1}$ and the sound speed is of the order of $1 \mathrm{~km} \mathrm{~s}^{-1}$ (for a temperature of the order of $80 \mathrm{~K}$, as inferred from the analysis of Sect. 3.3). The nature of the ring observed in the TX Psc image is unclear. Similar rings are clearly visible in the

\footnotetext{
${ }^{5}$ We stress, however, that Wareing et al. numerical models do not incorporate dust grains, and only handle gas warmer than $10^{4} \mathrm{~K}$ (because of the lack of an adequate cooling function for gas with lower temperatures). In such environments, dust grains would not be able to survive and therefore far-IR dust thermal emission is not expected. Although Fig. 7 shows structures resembling what is seen in PACS images, this model traces the hot gas density, which is not expected to emit the observed far-IR emission. Despite these shortcomings, the striking similarity beween the PACS and model images could be accounted for if the far-IR radiation comes from atomic emission lines in shocked gas. Alternatively, one may speculate that future models including the proper gas cooling function will find similar structures involving gas at lower temperatures, with dust naturally forming in regions of high gas densities and moderate temperatures.
}

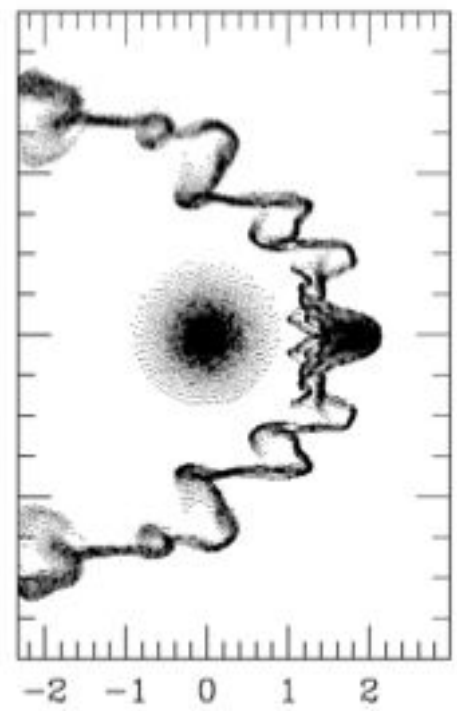

Fig. 8. The gas density in the Mach 10 simulation of Blondin \& Koerwer (1998). Note the strong blob upstream and the highly unstable and inhomogeneous bow shock. This case resembles the TX Psc nebula (compare with Fig. 6, remembering that the viewing angle is slightly different, since the space motion of TX Psc is inclined by $14^{\circ}$ with respect to the plane of the sky). Reprinted from Blondin \& Koerwer 1998, New Astron., 3, 571, with permission from Elsevier.
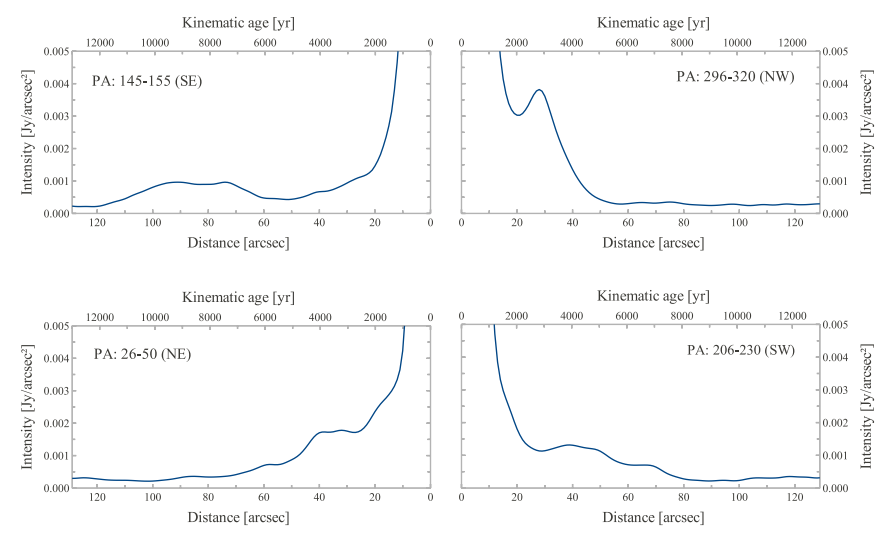

Fig. 9. Intensity cuts for X Her from the PACS $70 \mu \mathrm{m}$ deconvolved image (right panel of Fig. 6) along two perpendicular directions through the star, one corresponding to the space motion direction (upper panels). The dynamical ages on the upper scale correspond to a wind expansion velocity of $6.5 \mathrm{~km} \mathrm{~s}^{-1}$.

Wareing et al. simulations when the mass-losing star has reached the post-AGB phase (thus posterior to the stage displayed in Fig. 7). The ring thus corresponds to the planetary nebula shell. This cannot be the case for TX Psc, although the ring possibly traces a discrete episode of strong mass loss. Alternatively, it could be the termination shock.

\subsection{Interpreting the emission of the upstream blob}

As noticed at the end of Sect. 3.2, an upstream blob is clearly visible in the Mach-10 isothermal-shock simulations of Blondin \& Koerwer (1998) (Fig. 8), as well as in several of the simulations of Comerón \& Kaper (1998). Such a blob is present in the nebulae associated with both X Her and TX Psc. To investigate the 

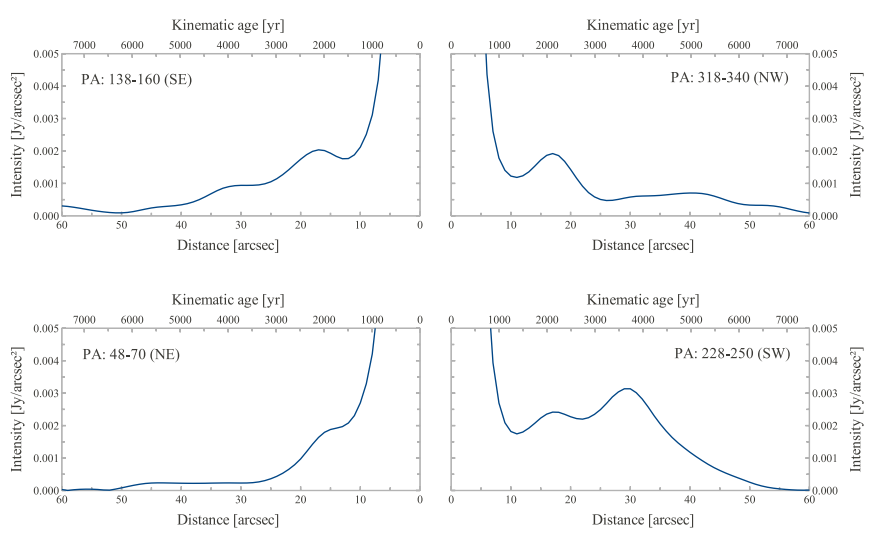

Fig. 10. Same as Fig. 9 for TX Psc and a wind expansion velocity of $10.5 \mathrm{~km} \mathrm{~s}^{-1}$.

nature of the emission associated with this blob, we performed aperture photometry in the 70 and $160 \mu \mathrm{m}$ bands, for TX Psc, where it is cleary detached from the surrounding material (lower right panel of Fig. 10). A radius of 5 arcsec was adopted to fully cover the blob. The background flux was estimated, from the periphery of the image, to be $3 \times 10^{-4}$ Jy per pixel at $70 \mu \mathrm{m}$ and $5 \times 10^{-4}$ Jy per pixel at $160 \mu \mathrm{m}$. With these values, we get total fluxes of $0.191 \mathrm{Jy}$ at $70 \mu \mathrm{m}$ and $0.039 \mathrm{Jy}$ at $160 \mu \mathrm{m}$, or a ratio $F_{v, 70} / F_{v, 160}=4.89$ (see also the lower panel of Fig. 11, which compares the intensity profiles in the 70 and $160 \mu \mathrm{m}$ bands). The observed light in this blob could in principle come from emission lines (such as [O I] $63 \mu \mathrm{m}$, [O I] $145 \mu \mathrm{m}$, and [C II] $158 \mu \mathrm{m}$ ) excited by the shock ${ }^{6}$. However, Spitzer/IRS spectroscopic observations of the R Hya bow shock did not detect atomic lines (Ueta 2011), and the detected far-IR radiation from the bow suggests instead the presence of very cold (20-40 K) material (Ueta et al. 2009). The same conclusion was reached by van Buren $\&$ McCray (1988) and Dgani et al. (1996a) in the context of OB runaway stars; they note that the dust trapped in the bow shock shell reradiates efficiently about $1 \%$ of the star's bolometric luminosity at far-infrared wavelengths.

Therefore, we make here the working hypothesis that the light emitted by the blob is from dust emission ${ }^{7}$, and derive the corresponding dust temperature. From Kirchhoff's law, we may write the dust emissivity $j_{v}$ as

$j_{v}=k_{v} j_{B B, v}(T)$

where $j_{B B, v}(T)$ is the black body emissivity at frequency $v$ and $k_{v}$ is the dust absorbing coefficient. Since TX Psc is a carbon star, we adopted the absorption coefficient for amorphous carbon as given by Draine (1981), who provides the $Q$ factors (ratio of the absorption cross section to the geometrical cross section) as a function of wavelength. Since $k_{v}$ is proportional to $Q$ and we are only interested in ratios, we may write

$\frac{F_{v, 70}}{F_{v, 160}}=\frac{Q_{70} j_{B B, v(70)}(T)}{Q_{160} j_{B B, v(160)}(T)}$,

\footnotetext{
${ }^{6}$ Remember that $\mathrm{H}_{\alpha}$ emission as well as UV radiation (caused by excited $\mathrm{H}_{2}$ molecules) are sometimes associated with AGB wind - ISM interaction, as in the case of Mira (Martin et al. 2007) and R Hya (Ueta 2008; Ueta et al. 2008)

7 Solving this issue would require the acquisition of a spectrum for this blob.
}
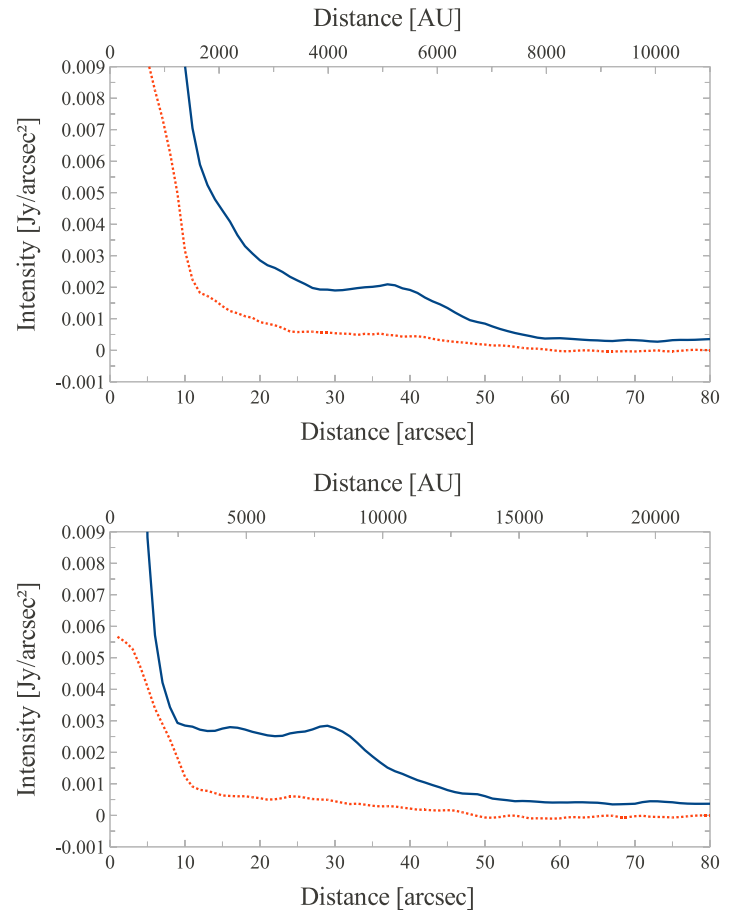

Fig. 11. Intensity plots for $X$ Her and TX Psc. Upper plot: integrated intensity of $\mathrm{X} \mathrm{Her}$ in the direction of the northern blob (PA: $-7^{\circ}$ to $-17^{\circ}$ ) from the image of Fig. 4. Lower plot: integrated intensity for TX Psc in direction of the blob/proper motion (PA: $228^{\circ}$ to $250^{\circ}$ ), from the image of Fig. 5. In both plots the (blue) continuous curve corresponds to $70 \mu \mathrm{m}$, the (red) dashed curve to $160 \mu \mathrm{m}$. The distances used to convert the angular scales into distances in AU are listed in Table 1.

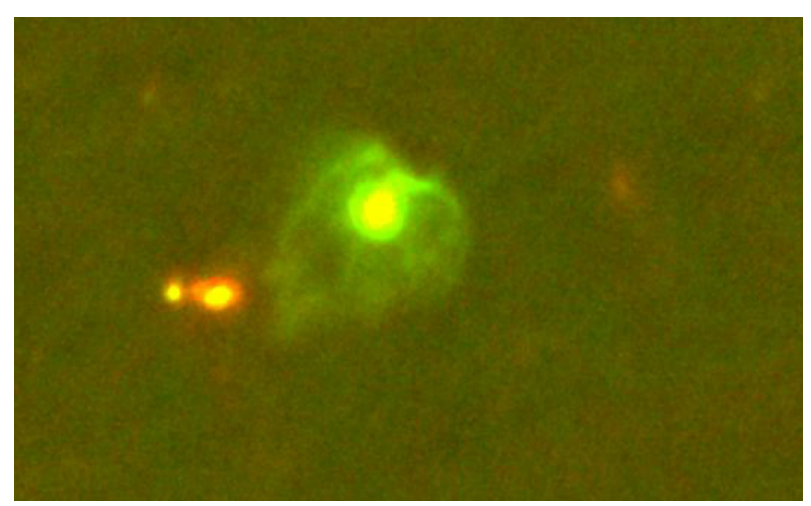

Fig. 12. Composite image merging the PACS 70 and $160 \mu \mathrm{m}$ images of $\mathrm{X}$ Her (respectively in the green and red channels).

where $Q_{70} / Q_{160}=512 / 219=2.34$. Thus the dust temperature is that satisfying the relation

$\frac{j_{B B, v(70)}(T)}{j_{B B, v(160)}(T)}=4.89 / 2.34=2.09$.

A temperature of $81 \mathrm{~K}$ for the blob satisfies the above condition. Since the absorption coefficient is for dust containing only amorphous carbon, the temperature derived above must be seen as a rough estimate. The same analysis for $\mathrm{X}$ Her, adopting the ratio $Q_{70} / Q_{160}=185 / 25=7.4$ for astronomical silicates (Draine 1985), yields a temperature of $40 \mathrm{~K}$. The composite colour image of X Her presented in Fig. 12 does not show evidence of any large temperature variations in the nebula, which is dominated by the emission at $70 \mu \mathrm{m}$. 


\subsection{Wilkin fitting of the bow shock interface}

The far-IR parabolic-like arc structures around X Her and TX Psc can be interpreted as the interface regions between the ISM and AGB winds, as already recognized for R Hya (Ueta et al. 2006), R Cas (Ueta et al. 2010), and IRC +10 216 (Ladjal et al. 2010). Assuming that (1) the ram-pressure balance is established between the ambient ISM and AGB winds and (2) momentum is conserved across a physically thin shock interface (i.e. the shock is radiatively cooled efficiently), one can express the bow shock shape analytically as a function of the latitudinal angle $\theta$ measured from the apex of the bow with respect to the position of the central star as formulated by Wilkin (1996)

$R(\theta)=R_{0} \frac{\sqrt{3(1-\theta \cot \theta})}{\sin \theta}$,

where $R_{0}$ is the stand-off distance between the star and bow apex, and defined as

$R_{0}=\sqrt{\frac{\dot{M} v_{\mathrm{w}}}{4 \pi \rho_{\mathrm{ISM}} v_{*, \mathrm{ISM}}^{2}}}$

for which $\dot{M}$ is the mass-loss rate, $v_{\mathrm{w}}$ is the isotropic stellar wind velocity, $\rho_{\text {ISM }}$ is the ambient ISM mass density, and $v_{*, \text { ISM }}$ is the relative velocity of the star with respect to the ambient ISM.

Because the observed surface brightness enhancement represents approximately a conic section of an inclined bow shock paraboloid by the plane of the sky intersecting the central star ${ }^{8}$, the inclination angle and deprojected stand-off distance of the bow shock paraboloid can (in principle) be determined by fitting the apparent shape of the bow shock using the analytical Wilkin function (the detailed description of the Wilkin fitting procedures may be found in Cox et al., in prep.).

As discussed in Sect. 3.2, the Herschel images of the targets at higher spatial resolution have resolved structures in the bow shock interface that resemble vortices caused by instabilities at the contact discontinuity (e.g., Wareing et al. 2007b). In particular, the X Her image shows that the bow shock interface is bent in the direction of the star (Fig. 6). This bent-interface structure appears typical of bow shocks caused by a large relative velocity between the star and the ambient ISM, $v_{*, \text { ISM }}$, as shown in hydrodynamical simulations by Wareing et al. (2007b, Fig. 7).

The presence of these bent structures obviously makes the application of the Wilkin fitting problematic. Nevertheless, we performed the Wilkin fitting of the bow shock with and without the bent region and obtained essentially the same results. While we present the results of Wilkin fitting below assuming that a conic section of the bow shock paraboloid is still recoverable from the overall shape of the observed far-IR surface brightness distributions, we recall that the observed bow shocks of X Her and TX Psc show asymmetric structures that were not seen in any previous AGB wind-ISM interaction cases.

Using the method outlined by Cox et al. (in preparation), the Wilkin fitting then yields a best-fit bow shock paraboloid with $R_{0}=36^{\prime \prime}$ (corresponding to $4930 \mathrm{AU}$ at the parallactic distance of $137 \mathrm{pc}$ ) at the position angle (PA; defined as degrees $\mathrm{E}$ of $\mathrm{N}$ ) of

\footnotetext{
8 Assuming the observed surface brightness is mainly due to thermal dust emission, the surface brightness is roughly proportional to the dust column density along the line of sight in the optically thin limit, which is true for the far-IR wavelengths. If the dust distribution is an inclined paraboloid, the column density becomes the greatest at the intersection of the structure with the plane of the sky and therefore the observed surface brightness distribution represents the conic section of the paraboloid.
}

$330^{\circ}$ for X Her and with $R_{0}=37^{\prime \prime}$ (corresponding to $10175 \mathrm{AU}$ at the parallactic distance of $275 \mathrm{pc}$ ) and at the PA of $238^{\circ}$ for TX Psc.

The PA obtained above (characterizing the relative motion of the AGB star with respect to the ambient ISM as determined from the apparent heliocentric orientation of the bow shock paraboloid, and denoted $v_{*, \text { ISM }}$ ) must be compared with the PA of the stellar space motion in the LSR $\left(v_{*, \mathrm{LSR}}\right)$, as listed in Table 1. They are, for both stars, roughly consistent with each other: $330^{\circ}$ and $309^{\circ}$ for $X$ Her, and $238^{\circ}$ and $243^{\circ}$ for TX Psc. The $20^{\circ}$ discrepancy observed for X Her may be an indication that the ISM around that star is not at rest in the LSR; however, we do not attempt to infer the velocity of that ISM flow, because of the uncertainties associated with Wilkin fitting for such peculiar shock shapes as observed around the two stars under consideration.

Assuming as a first approximation that the ISM is at rest in the LSR, (thus $v_{*, \mathrm{ISM}}=v_{*, \mathrm{LSR}}$, the latter value being listed in Table 1), Eq. (5) may then be used to derive the ISM density close to X Her and TX Psc, knowing their mass loss rate and wind velocity. Adopting for these values $\dot{M}=1.5 \times 10^{-7} M_{\odot} \mathrm{yr}^{-1}$ and $6.5 \mathrm{~km} \mathrm{~s}^{-1}$ for X Her (González-Delgado et al. 2003) and $\dot{M}=9.1 \times 10^{-8} M_{\odot} \mathrm{yr}^{-1}$ and $10.5 \mathrm{~km} \mathrm{~s}^{-1}$ for TX Psc (Olofsson et al. 1993), we find $n_{\mathrm{H}}=0.67$ and 0.16 atoms $\mathrm{cm}^{-3}$ for X Her and TX Psc, respectively. These values appear quite reasonable, given the high Galactic latitudes of these two stars (Table 1).

\section{Bipolar outflows and global picture}

\section{1. $X \mathrm{Her}$}

An extensive description of the circumstellar environment of X Her can be found in Gardan et al. (2006) and Matthews et al. (2011), and only the most noteworthy features will be repeated here. X Her has distinctly double-component lines in $\mathrm{CO}(J=2-1)$ and $(J=3-2)$, i.e., a narrow feature centred on a broader feature, with $v_{\mathrm{w}} \sim 3$ and $\sim 9 \mathrm{~km} \mathrm{~s}^{-1}$, respectively (Kahane \& Jura 1996; Knapp et al. 1998; Kerschbaum \& Olofsson 1999; Olofsson et al. 2002; González-Delgado et al. 2003; Nakashima 2005; Castro-Carrizo et al. 2010). GonzálezDelgado et al. (2003) observe a similarly double-component $\mathrm{SiO}(v=0, J=2-1)$ line; from $\mathrm{CO}$ data, they derive mass-loss rates of $1.5 \times 10^{-7} M_{\odot} \mathrm{yr}^{-1}$ and $0.4 \times 10^{-7} M_{\odot} \mathrm{yr}^{-1}$, respectively, for the broad and narrow components. Although Knapp et al. (1998) argued that this complex line profile may be caused by episodic mass loss with highly varying gas expansion velocities, it seems more likely now that it results from a complicated flow geometry.

The spatial information provided by the $\mathrm{CO}$ radio line observations of Kahane \& Jura (1996) suggests that the broad plateau is a bipolar outflow oriented in the SW-NE direction, with the SW side facing towards us, a structure that was confirmed by Nakashima (2005). The most recent CO radio line interferometry data of Castro-Carrizo et al. (2010) clearly resolves an hour-glass structure in the higher-velocity emission with a position angle of $45^{\circ}$, i.e., this indicates that a collimated bipolar wind in this direction has shaped the original AGB circumstellar envelope. The inclination angle of the outflow is likely to be small. Castro-Carrizo et al. (2010) find no evidence of a (rotating) disk perpendicular to the outflow axis. We obtained $\mathrm{SiO}$ $(v=0, J=1-0)$ line data towards X Her using the Very Large Array (details about these data and their reduction are given in the Appendix). These data probe a region smaller than that of the $\mathrm{CO}$ data because the $\mathrm{SiO}$ molecules are confined to a smaller region and they are effectively excited only in the inner more dense 


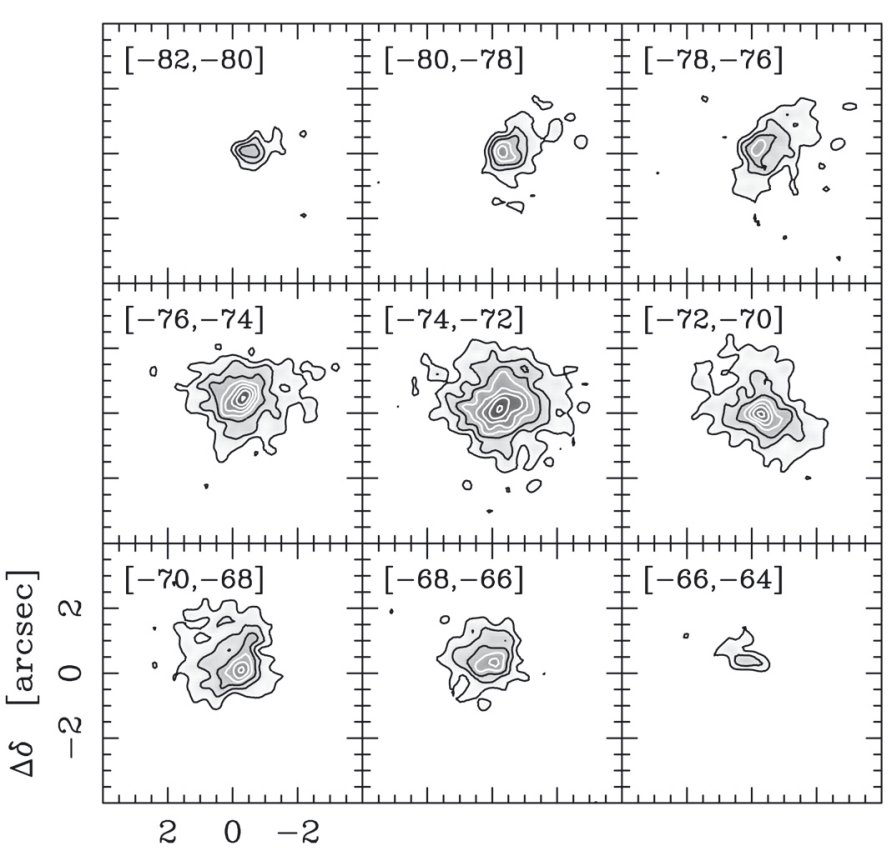

$\Delta \alpha \quad[\operatorname{arcsec}]$

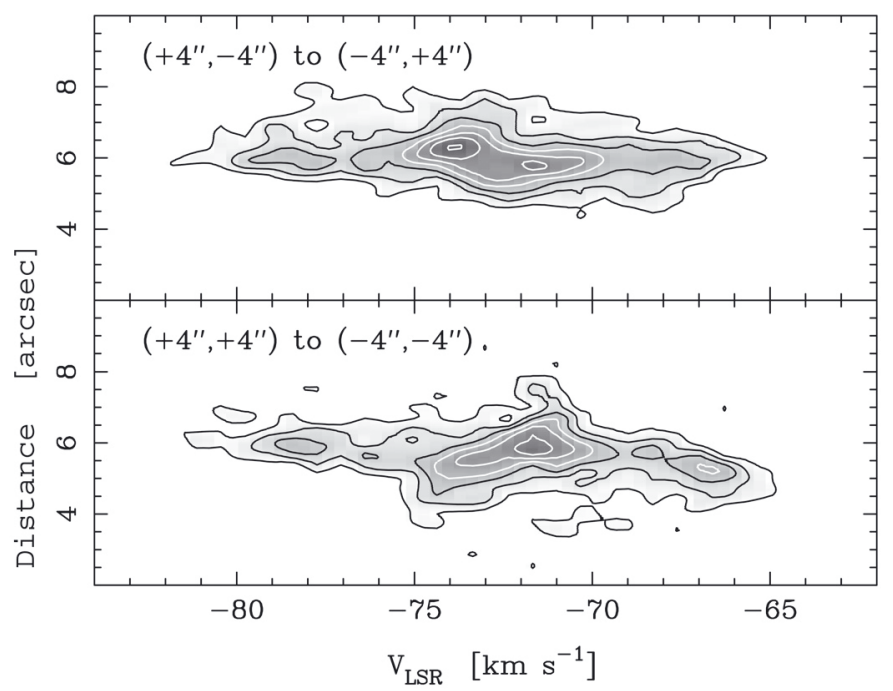

Fig. 13. Maps from the Very Large Array in the $\mathrm{SiO}(v=0, J=1-0)$ line for X Her. The upper panels show $2 \mathrm{~km} \mathrm{~s}^{-1}$ wide channel maps, and the lower panels show position (in arcsecs, along the vertical axis) - velocity (in $\mathrm{km} \mathrm{s}^{-1}$, along the horizontal axis) diagrams along the NE-SW [labelled $(+4,+4)$ to $(-4,-4)$ ] and NW-SE [labelled $(+4,-4)$ to $(-4$, $+4)$ ] directions. Velocities are with respect to the local standard of rest (systemic velocity of X Her is $-72.5 \mathrm{~km} \mathrm{~s}^{-1}$ ). The lower panels show evidence of a bipolar outflow in the NE-SW direction (thus perpendicular to the space motion) and of a rotating disk in the NW-SE direction. The contour increments are $10 \mathrm{mJy} \mathrm{km} \mathrm{s}{ }^{-1}$ per beam for the channel maps in the upper panel and $5 \mathrm{mJy}$ per beam for the position-velocity diagrams in the lower panel. The beam size is $0.45^{\prime \prime} \times 0.40^{\prime \prime}$ (with a position angle of $-88 \mathrm{deg}$ ).

parts of the circumstellar envelope. Nevertheless, the channel map data presented in Fig. 13 show a structure very similar to that of the $\mathrm{CO}$ data, a bipolar outflow at position angle $\approx 45^{\circ}$ and a hint of an hour-glass structure. The position-velocity diagram along the outflow axis is also very similar to that obtained from the CO line data (Fig. 13). Interestingly, the position-velocity diagram perpendicular to this axis shows a structure that could be interpreted in terms of a rotating disk.
Gardan et al. (2006) and Matthews et al. (2011) detected the $\mathrm{H} \mathrm{I}$ emission at $21 \mathrm{~cm}$ from the circumstellar shell around X Her, and found it to be extended along the direction of the space motion ( $\geq 6^{\prime}$ or about $0.24 \mathrm{pc}$ ). Matthews et al. (2011) found, in their $\mathrm{H}$ I data and on an arcmin scale, some evidence for the continuation of the bipolar outflow observed on small scales $\left( \pm 10^{\prime \prime}\right)$ in the molecular $\mathrm{CO}$ and $\mathrm{SiO}$ lines.

The surprising picture that emerges from the combination of all the data, including the new Herschel data set, is that of a bipolar outflow in the innermost region $\left( \pm 10^{\prime \prime}\right)$, that appears to be almost exactly perpendicular to the far-IR and H I nebula oriented along the direction of space motion and caused by the interaction between the stellar wind and the ISM. It is not at all clear whether this is just a chance coincidence, or whether there could be a causal relationship between the orientation of the bipolar outflow and the space motion. The absence of any sign whatsoever of the bipolar outflow visible in the Herschel images would tend to dismiss the latter possibility. The existence of a bipolar outflow seen in $\mathrm{CO}$ and $\mathrm{SiO}$ molecular lines raises another question: how can this outflow be reconciled with the bow shock seen all around the star and signaling that mass loss is (was?) roughly isotropic? Two solutions may be envisioned: (i) even in the presence of a bipolar outflow, mass continues to be ejected in all directions, albeit probably at a lower rate; (ii) the bipolar outflow has formed more recently than the features seen in the far IR.

\section{2. $T X P S C$}

The close environment of TX Psc, as probed by the molecular lines, appears to be quite complex. The observed line profiles of the CO line emission towards TX Psc are indeed peculiar, as first noted by Heske et al. (1989). As a consequence, the expansion velocity derived from the observed radio line profiles range from 7.5 to $12.2 \mathrm{~km} \mathrm{~s}^{-1}$ (see Table 2 of Schöier \& Olofsson 2001; and Loup et al. 1993) depending on the transition and the telescope used. Heske et al. (1989) mapped the circumstellar CO line emission around this object and interpreted the observed asymmetries as being produced by either a bipolar outflow or a highly clumped wind. No detailed modelling exists for the circumstellar shell around TX Psc, and its evolutionary status remains uncertain.

The asymmetry detected by Heske et al. (1989) and possibly attributable to a bipolar outflow is oriented along the NESW direction, with the red- and blue-shifted part of the emission separated by about 10 arcsec. However, there is some ambiguity about this direction in the original paper. Interestingly enough, both lunar occultation data (Richichi et al. 1995) and Come-On+ adaptive-optics observations (Cruzalèbes et al. 1998) claim as well that the source is asymmetric. The September 1994 ComeOn+ observations in the $K$ band clearly reveal a secondary source $0.35^{\prime \prime}$ to the SW (with a position angle of $241^{\circ}$ ) of the primary source, with a flux ratio of $2 \%$. The lunar occultation observations indicate that the brightness profile of TX Psc has a structure that becomes increasingly more complex when going from the $V$ band to the $L$ band, where it is double peaked (on an angular scale of a few milliarcsecs). The $V$ band reveals some secondary peaks on either side of the central core. In the visual band, Hartkopf et al. (1996) and Mason et al. (1999) could not, however, resolve TX Psc between 1993 and 1997 with a resolving limit of $0.054^{\prime \prime}$.

The gas mass-loss rate of TX Psc has been derived by several authors. Olofsson et al. (1993) found a very moderate gas massloss rate of $9.1 \times 10^{-8} M_{\odot} \mathrm{yr}^{-1}$ from CO observations and a wind 
expansion velocity of $10.5 \mathrm{~km} \mathrm{~s}^{-1}$. Loup et al. (1993) found $5.6 \times$ $10^{-7} M_{\odot} \mathrm{yr}^{-1}$ and in a later paper Schöier \& Olofsson (2001) derived an expansion velocity of $7.5 \mathrm{~km} \mathrm{~s}^{-1}$, with a complex $\mathrm{CO}$ line shape that prevented them from attempting to derive the mass-loss rate.

The new Herschel/PACS data described in Sect. 3 confirm the presence of an axisymmetry in the far IR with a NE-SW axis and on an arcmin scale. However, in contrast to the situation prevailing for X Her, the asymmetries observed around TX Psc on an arcsec scale have the same orientation as the proper motion and the far-IR nebula. Nevertheless, an almost circular shell, with a radius of about 17", surrounds TX Psc and does not bear any signature of the asymmetries observed on a much smaller angular scale.

\section{Conclusions}

The PACS images of the O-rich star X Her and the C-rich star TX Psc obtained by Herschel at 70 and $160 \mu \mathrm{m}$ reveal a complex structure with signatures of interaction between the AGB wind and the ISM. Their space velocities, corrected from the solar motion, are 90 and $67 \mathrm{~km} \mathrm{~s}^{-1}$, respectively. Its velocity locates $\mathrm{X}$ Her among the high-velocity group defined by Famaey et al. (2005) in their kinematic study of giant stars from the Hipparcos Catalogue. The most noteworthy feature is, for both stars, the presence of a clump located right along the direction of the space motion. It is very likely the signature of instabilities arising in the shock front between the ISM and the AGB wind. The kinematical age of these clumps is (at least) about 2000 yrs for both stars. Although emission lines excited by the shock may contribute to the observed infrared light, assuming instead that only dust emission is involved yields a dust temperature of about $40 \mathrm{~K}$ for X Her and $80 \mathrm{~K}$ for TX Psc. The definite identification of the physical process at the origin of the light emitted by the bright clumps must await their detailed spectroscopic analysis. Further downstream along the bow shock, the PACS images reveal the presence of fainter clumps that may be associated with KelvinHelmholtz vortices peeling off the bow shock and moving downstream. There is actually a striking similarity between the PACS image of X Her, and the predictions of the hydrodynamical simulation of Wareing et al. (2007a) for the case $v_{* \text { ISM }}=125 \mathrm{~km} \mathrm{~s}^{-1}$ (as compared to the observed value of $90 \mathrm{~km} \mathrm{~s}^{-1}$ ), $n_{\mathrm{H}}=2 \mathrm{~cm}^{-3}$, and a mass-loss rate of $5 \times 10^{-6} M_{\odot} \mathrm{yr}^{-1}$ (a factor of 30 larger than the current mass loss rate, however), where a front shock is bent towards the mass-losing star along the upstream direction, and K-H vortices are downstream along the bow shock. The strong blob visible upstream of TX Psc may be an indication that the wind and the ISM flow are highly supersonic, according to the simulations of Blondin \& Koerwer (1998).

The Wilkin fitting of the bow shock shape is made difficult by its disturbed, highly structured shape (K-H instabilities?) and its reverse bending centred on the upstream direction. Nonetheless, the position angle of the bow shock obtained by a Wilkin fitting is roughly consistent with the position angle of the space motion of the star with respect to the LSR (the largest discrepancy is $20^{\circ}$ for X Her), indicating that the ISM is not far from being at rest in the LSR local to the stars.

A very puzzling conclusion of the present analysis is that for both stars the axisymmetry caused by the space motion and its interaction with the ISM seems to be maintained down to very small angular scales, even beyond the circular feature present on intermediate scales in both TX Psc and X Her:

- In X Her, a secondary source almost perfectly aligned with the space motion was discovered by earlier COME-ON+ observations, despite being located well within the ring observed with PACS.

- Around TX Psc, a bipolar outflow extending over a few arcseconds has been reported by radio observations (with some ambiguity however, about its orientation, but seemingly parallel to the space motion). A bipolar outflow was also reported for X Her, and for that star, the space motion and the outflow are clearly perpendicular to each other.

The existence of those correlations between the axisymmetry present in the inner and outer circumstellar regions of TX Psc and X Her (despite circular structures being present on intermediate scales, possibly associated with the termination shock) is the most puzzling conclusion from the present work, for which we do not currently have any convincing explanation. Similarly, it is rather surprising that the far-IR nebula contains a nearly perfectly circular shell (either interior to or identical to the termination shock), indicative of (past) isotropic mass loss, whereas there is at the same time an inner bipolar outflow. Could this be an indication that the mass loss became bipolar quite recently?

Acknowledgements. We acknowledge with thanks the variable star observations from the AAVSO International Database contributed by observers worldwide and used in this research. This work was supported in part by the Belgian Federal Science Policy Office via the PRODEX Programme of ESA (No. C90370). W.N. acknowledges funding by the FWF under project number P21988-N16. F.K. acknowledges funding by the Austrian Science Fund FWF under project number P23586-N16, R.O. under project number I163-N16, J.H. under project number P19503-N16. The Very Large Array is operated by the National Radio Astronomy Observatory, which is a facility of the National Science Foundation operated under cooperative agreement by Associated Universities, Inc.

\section{Appendix A: SiO line VLA observations of X Her}

$\mathrm{X}$ Her was observed in the rotational lines of SiO using the Very Large Array in C configuration. As part of a 10-h observing run on 2001 August 24, about one hour was spent to observe the $(J=1-0, v=0)$ transition at $43.424 \mathrm{GHz}$ in dual circular polarization. A long run of nine hours in right-hand circular polarization (RCP) was observed on 2004 March 2004. We combined these data with an additional hour dual polarization observations taken on 2004 April 22.

All these observations used a total bandwidth of $6.25 \mathrm{MHz}$ $\left(\sim 40 \mathrm{~km} \mathrm{~s}^{-1}\right)$ centred on a Doppler velocity of $-71 \mathrm{~km} \mathrm{~s}^{-1}$ with respect to the Local Standard of Rest. The bandwidth was divided in 64 spectral channels, corresponding to a channel separation of $\sim 0.67 \mathrm{~km} \mathrm{~s}^{-1}(97.7 \mathrm{kHz})$, for all three observation runs. Also common to the observations was the fast switching mode to the phase reference source $\mathrm{J} 1549+5038$ using a typical cycle of $\sim 40$ and $100 \mathrm{~s}$ for calibration and target source. Using 3C286 as a flux calibrator, we measured fluxes for J1549+5038 of 0.52 $( \pm 0.01)$ and $0.61( \pm 0.01)$ Jy for 2001 August and 2004 March, respectively. We set it to be 0.62 Jy for the 2004 April observations.

The data were reduced using standard editing, calibration, and imaging procedures available in the Astronomical Image Processing System. The RCP data was combined with the dual polarization data to Stokes I, assuming that the thermal radiation is unpolarized (i.e., Stokes $V=0$ ). The concatenated visibility data set was imaged into a cleaned cube of $\sim 13^{\prime \prime} \times 13^{\prime \prime}$ over -87 to $-54 \mathrm{~km} \mathrm{~s}^{-1}$ with an angular resolution of about $0.5^{\prime \prime}$ and a RMS noise of typically $2.2 \mathrm{mJy}$ per channel. The results of these observations are displayed in Fig. 13. 


\section{References}

Barnbaum, C., \& Hinkle, K. H. 1995, AJ, 110, 805

Blondin, J. M., \& Koerwer, J. F. 1998, New A, 3, 571

Blondin, J. M., \& Marks, B. S. 1996, New A, 1, 235

Borkowski, K. J., Sarazin, C. L., \& Soker, N. 1990, ApJ, 360, 173

Castro-Carrizo, A., Neri, R., Winters, J. M., et al. 2007, in Why Galaxies

Care About AGB Stars: Their Importance as Actors and Probes, ed.

F. Kerschbaum, C. Charbonnel, \& R. F. Wing, ASP Conf. Ser., 378, 199

Castro-Carrizo, A., Quintana-Lacaci, G., Neri, R., et al. 2010, A\&A, 523, A59

Comerón, F., \& Kaper, L. 1998, A\&A, 338, 273

Cruzalèbes, P., Lopez, B., Bester, M., Gendron, E., \& Sams, B. 1998, A\&A, 338, 132

Davis, D. S., Richer, H. B., King, I. R., et al. 2008, MNRAS, 383, L20

Dgani, R., \& Soker, N. 1998, ApJ, 495, 337

Dgani, R., van Buren, D., \& Noriega-Crespo, A. 1996a, ApJ, 461, 927

Dgani, R., van Buren, D., \& Noriega-Crespo, A. 1996b, ApJ, 461, 372

Draine, B. T. 1981, ApJ, 245, 880

Draine, B. T. 1985, ApJS, 57, 587

Eriksson, K., Gustafsson, B., Johnson, H. R., et al. 1986, A\&A, 161, 305

Famaey, B., Jorissen, A., Luri, X., et al. 2005, A\&A, 430, 165

Fregeau, J. M., Richer, H. B., Rasio, F. A., \& Hurley, J. R. 2009, ApJ, 695, L20

Freytag, B., \& Höfner, S. 2008, A\&A, 483, 571

Gardan, E., Gérard, E., \& Le Bertre, T. 2006, MNRAS, 365, 245

Geise, K. M., Ueta, T., Speck, A. K., Izumiura, H., \& Stencel, R. E. 2010, BAAS, 42, 364

González-Delgado, D., Olofsson, H., Kerschbaum, F., et al. 2003, A\&A, 411, 123

Groenewegen, M. A. T., Waelkens, C., Barlow, M., et al. 2011, A\&A, 526, A162 Gull, T. R., \& Sofia, S. 1979, ApJ, 230, 782

Hartkopf, W. I., Mason, B. D., McAlister, H. A., et al. 1996, AJ, 111, 936

Heske, A., te Lintel Hekkert, P., \& Maloney, P. R. 1989, A\&A, 218, L5

Heyl, J. 2007a, MNRAS, 381, L70

Heyl, J. 2007b, MNRAS, 382, 915

Heyl, J. S. 2008, MNRAS, 385, 231

Hinkle, K. H., Lebzelter, T., Joyce, R. R., \& Fekel, F. C. 2002, AJ, 123, 1002

Huggins, P. J., Mauron, N., \& Wirth, E. A. 2009, MNRAS, 396, 1805

Izumiura, H., Ueta, T., Yamamura, I., et al. 2011, A\&A, 528, A29

Jacoby, G. H. 1981, ApJ, 244, 903

Johnson, D. R. H., \& Soderblom, D. R. 1987, AJ, 93, 864

Kahane, C., \& Jura, M. 1996, A\&A, 310, 952

Kerschbaum, F., \& Olofsson, H. 1999, A\&AS, 138, 299

Kerschbaum, F., Ladjal, D., Ottensamer, R., et al. 2010, A\&A, 518, L140

Kerschbaum, F., Mecina, M., Ottensamer, R., et al. 2011, in Why galaxies care about AGB stars, ed. T. Lebzelter, ASP Conf. Ser., in press

Knapp, G. R., Young, K., Lee, E., \& Jorissen, A. 1998, ApJS, 117, 209

Krautter, J., Radons, G., \& Klaas, U. 1987, A\&A, 181, 373

Kwok, S., Volk, K., \& Bidelman, W. P. 1997, ApJS, 112, 557

Ladjal, D., Barlow, M. J., Groenewegen, M. A. T., et al. 2010, A\&A, 518, L141

Lebzelter, T., Hinkle, K. H., \& Aringer, B. 2001, A\&A, 377, 617

Libert, Y., Le Bertre, T., Gérard, E., \& Winters, J. M. 2008, A\&A, 491, 789

Libert, Y., Winters, J. M., Le Bertre, T., Gérard, E., \& Matthews, L. D. 2010, A\&A, 515, A112

Loup, C., Forveille, T., Omont, A., \& Paul, J. F. 1993, A\&AS, 99, 291

Martin, D. C., Seibert, M., Neill, J. D., et al. 2007, Nature, 448, 780

Martin, J., Xilouris, K., \& Soker, N. 2002, A\&A, 391, 689

Mason, B. D., Martin, C., Hartkopf, W. I., et al. 1999, AJ, 117, 1890

Mastrodemos, N., \& Morris, M. 1999, ApJ, 523, 357

Matt, S., Balick, B., Winglee, R., \& Goodson, A. 2000, ApJ, 545, 965
Matthews, L. D., \& Reid, M. J. 2007, AJ, 133, 2291

Matthews, L. D., Libert, Y., Gérard, E., Le Bertre, T., \& Reid, M. J. 2008, ApJ, 684,603

Matthews, L. D., Libert, Y., Gérard, E., et al. 2011, AJ, 141, 60

Mauron, N., \& Huggins, P. J. 2000, A\&A, 359, 707

Mauron, N., \& Huggins, P. J. 2006, A\&A, 452, 257

Meixner, M., Ueta, T., Dayal, A., et al. 1999, ApJS, 122, 221

Morris, M., Sahai, R., Matthews, K., et al. 2006, in Planetary Nebulae in our Galaxy and Beyond, ed. M. J. Barlow, \& R. H. Méndez, IAU Symp., 234, 469

Nakashima, J. 2005, ApJ, 620, 943

Needham, C. E. 2010, Blast waves (New York: Springer)

Nicholls, C. P., Wood, P. R., Cioni, M., \& Soszyński, I. 2009, MNRAS, 399, 2063

Olofsson, H., Eriksson, K., Gustafsson, B., \& Carlstrom, U. 1993, ApJS, 87, 267

Olofsson, H., González-Delgado, D., Kerschbaum, F., \& Schöier, F. L. 2002, A\&A, 391, 1053

Ottensamer, R., Luntzer, A., Mecina, M., et al. 2011, in Why galaxies care about AGB stars II, ed. T. Lebzelter, ASP Conf. Ser., in press

Raskin, G., Van Winckel, H., Hensberge, H., et al. 2010, A\&A, 526, A69

Reimers, C. 2005, in Ph.D. thesis, University of Vienna

Reynolds, R. J. 1985, ApJ, 288, 622

Richichi, A., Chandrasekhar, T., Lisi, F., et al. 1995, A\&A, 301, 439

Sahai, R., \& Chronopoulos, C. K. 2010, ApJ, 711, L53

Schöier, F. L., \& Olofsson, H. 2001, A\&A, 368, 969

Schönrich, R., Binney, J., \& Dehnen, W. 2010, MNRAS, 403, 1829

Sloan, G. C., Levan, P. D., \& Little-Marenin, I. R. 1996, ApJ, 463, 310

Sloan, G. C., Kraemer, K. E., Goebel, J. H., \& Price, S. D. 2003, ApJ, 594, 483

Soker, N. 2005, in Planetary Nebulae as Astronomical Tools, ed. R. Szczerba, G. Stasińska, \& S. K. Gorny, AIP Conf. Ser., 804, 89

Soker, N., Borkowski, K. J., \& Sarazin, C. L. 1991, AJ, 102, 1381

Stencel, R. E., Carpenter, K. G., Pesce, J. E., et al. 1988, ESA SP-281, 249

Theuns, T., \& Jorissen, A. 1993, MNRAS, 265, 946

Theuns, T., Boffin, H. M. J., \& Jorissen, A. 1996, MNRAS, 280, 1264

Ueta, T. 2008, ApJ, 687, L33

Ueta, T. 2011, in Why galaxies care about AGB stars II, ed. T. Lebzelter, ASP Conf. Ser., in press

Ueta, T., Meixner, M., \& Bobrowsky, M. 2000, ApJ, 528, 861

Ueta, T., Speck, A. K., Stencel, R. E., et al. 2006, ApJ, 648, L39

Ueta, T., Izumiura, H., Yamamura, I., et al. 2008, PASJ, 60, 407

Ueta, T., Stencel, R. E., Yamamura, I., et al. 2009, in AKARI, a Light to Illuminate the Misty Universe, ed. T. Onaka, G. J. White, T. Nakagawa, \& I. Yamamura, ASP Conf. Ser., 418, 463

Ueta, T., Stencel, R. E., Yamamura, I., et al. 2010, A\&A, 514, A16

van Buren, D. 1993, in Massive Stars: Their Lives in the Interstellar Medium, ed. J. P. Cassinelli, \& E. B. Churchwell, ASP Conf. Ser., 35, 315

van Buren, D., \& McCray, R. 1988, ApJ, 329, L93

van Buren, D., Noriega-Crespo, A., \& Dgani, R. 1995, AJ, 110, 2914

van der Veen, W. E. C. J., \& Habing, H. J. 1988, A\&A, 194, 125

van Leeuwen, F. 2007, A\&A, 474, 653

Villaver, E., García-Segura, G., \& Manchado, A. 2003, ApJ, 585, L49

Vishniac, E. T. 1994, ApJ, 428, 186

Wareing, C. J., Zijlstra, A. A., \& O'Brien, T. J. 2007a, MNRAS, 382, 1233

Wareing, C. J., Zijlstra, A. A., \& O'Brien, T. J. 2007b, ApJ, 660, L129

Wasatonic, R. 1995, Inf. Bull. Var. Stars, 4159, 1

Wasatonic, R. P. 1997, J. Am. Assoc. Var. Star Obs., 26, 1

Wilkin, F. P. 1996, ApJ, 459, L31

Wirsich, J. 1991, ApJ, 377, 285

Young, K., Phillips, T. G., \& Knapp, G. R. 1993, ApJS, 86, 517 